\title{
Complex-Coefficient Frequency Domain Stability Analysis Method for a Class of Cross-Coupled Antisymmetrical Systems and Its Extension in MSR Systems
}

\author{
Yuan Ren ${ }^{1,2}$ and Jiancheng Fang ${ }^{2}$ \\ ${ }^{1}$ Department of Space Equipment, Equipment Academy, Beijing 101416, China \\ ${ }^{2}$ School of Instrumentation Science \& Opto-electronics Engineering, Beihang University, Beijing 100191, China \\ Correspondence should be addressed to Yuan Ren; renyuan_823@aliyun.com
}

Received 25 July 2013; Revised 13 December 2013; Accepted 16 December 2013; Published 20 February 2014

Academic Editor: Asier Ibeas

Copyright ( $\odot 2014$ Y. Ren and J. Fang. This is an open access article distributed under the Creative Commons Attribution License, which permits unrestricted use, distribution, and reproduction in any medium, provided the original work is properly cited.

\begin{abstract}
This paper develops a complex-coefficient frequency domain stability analysis method for a class of cross-coupled two-dimensional antisymmetrical systems, which can greatly simplify the stability analysis of the multiple-input multiple-output (MIMO) system. Through variable reconstruction, the multiple-input multiple-output (MIMO) system is converted into a single-input single-output (SISO) system with complex coefficients. The pole locations law of the closed-loop system after the variable reconstruction has been revealed, and the controllability as well as observability of the controlled plants before and after the variable reconstruction has been studied too, and then the classical Nyquist stability criterion is extended to the complex-coefficient frequency domain. Combined with the rigid magnetically suspended rotor (MSR) system with heavy gyroscopic effects, corresponding stability criterion has been further developed. Compared with the existing methods, the developed criterion for the rigid MSR system not only accurately predicts the absolute stability of the different whirling modes, but also directly demonstrates their relative stability, which greatly simplifies the analysis, design, and debugging of the control system.
\end{abstract}

\section{Introduction}

The cross-coupling effect exists widely in many systems in practice, for example, the magnetically suspended rotor (MSR) systems [1-4], spacecraft attitude control systems [4, 5], power electronic systems [6-9], optical systems [10, 11], and so on [12].

Commonly, the cross-coupled systems can be classified into two kinds: the cross-coupled symmetrical and antisymmetrical systems $[13,14]$. As for the controlled plant with antisymmetrical cross-coupling characteristics, antisymmetrical cross-decoupling controller is often needed to realize the decoupling control of the system. This paper focuses on the stability analysis of the cross-coupled two-dimensional antisymmetrical system. For the sake of simplicity and clarity, the whole control system including the cross-coupled antisymmetrical controlled plant and its antisymmetrical crossdecoupling controller is called the cross-coupled antisymmetrical system in this paper.
Cross-coupled antisymmetrical system is a class of special multi-input multi-output (MIMO) systems. As for the stability analysis of the MIMO system, the most traditional method resorts to resolve the characteristic roots [15]. However, as for the high-order cross-coupled antisymmetrical system, it is very tedious and even impossible to obtain its analytical expressions. Although this issue may be alleviated by employing the Routh-Hurwitz criterion [16-18], this method cannot present the whole information about the system stability. Additionally, rebuilding a proper Lyapunov function is not easy since there is no rule to follow [19], especially for a highorder MIMO system. Therefore, when employed to judge the stability of the cross-coupled antisymmetrical system, these traditional methods are relatively complex. Moreover, the resolved relative stability information is abstract since they lack specific physical meanings. As a result, these methods have some difficulty in combining the stability analysis with the controller design. 
Accordingly, it is quite interesting and significative to judge directly and simply the stability of the cross-coupled antisymmetrical system, which is also a basic theoretical issue to be urgently addressed in engineering applications. An ideal stability criterion must be combined tightly with the design process of the controller, which can effectively guide the system design and further reveal the influence of the variation of the system parameters on the system stability [20]. Obviously, the above methods can not satisfy this requirement, and it will be more difficult when considering simultaneously the variations of the multiple system parameters.

The Nyquist stability criterion of the single-variable system has many advantages [21-24]. It not only can judge the closed-loop stability, but also can directly reveal the relationships between the relative stability of the system and its gain. Although this method has been extended to the multivariable case [25], such as characteristic locus method [26-31] and the inverse Nyquist array (INA) method [32, 33], these extended methods are quite complex since they depend on some special computer-aided-design (CAD) softwares. Especially when diagonal dominance does not hold, the stability of the MIMO system cannot be judged by the INA method.

Among all the cross-coupled antisymmetrical systems, the MSR system is a class of typical cases in which stability analysis is quite important since the MSR system is an openloop unstable system with high rotor speed [34-36]. Especially for the MSR system with significant gyroscopic effects, its whirling modes stability analysis is more challenging. However, the existing stability analysis methods [37-40] can only judge the stability of the whole whirling modes and cannot present the stability information of the different whirling modes, which is the key concern of the controller design and debugging. To solve this issue, the whirling modes stability criterion [41] based on complex coefficient frequency characteristics has been proposed, which can judge the stability of the different whirling modes. This method is of great value to the development of stability analysis of control theory. However, this method is only suited to the special MSR system, and it does not suit a class of cross-coupled antisymmetrical systems. How to extend the method to a class of cross-coupled systems is a great interest in engineering.

To simplify the absolute stability judgment of the crosscoupled antisymmetrical system and reveal directly its relative stability, a complex-coefficient frequency domain stability analysis method for a class of cross-coupled antisymmetrical systems is presented in this paper.

\section{Complex-Coefficient Frequency Domain Stability Analysis Method}

2.1. Variable Reconstruction of the Cross-Coupled Antisymmetric System. Figure 1 shows the structural scheme of the crosscoupled two-dimensional antisymmetrical system, where $K_{1}(s)=K_{2}(s)=K(s), T_{d 1}(s)=T_{d 2}(s)=T_{d}(s), T_{c r 1}(s)=$ $T_{c r 2}(s)=T_{c r}(s)$. Without loss of generality, the standard type

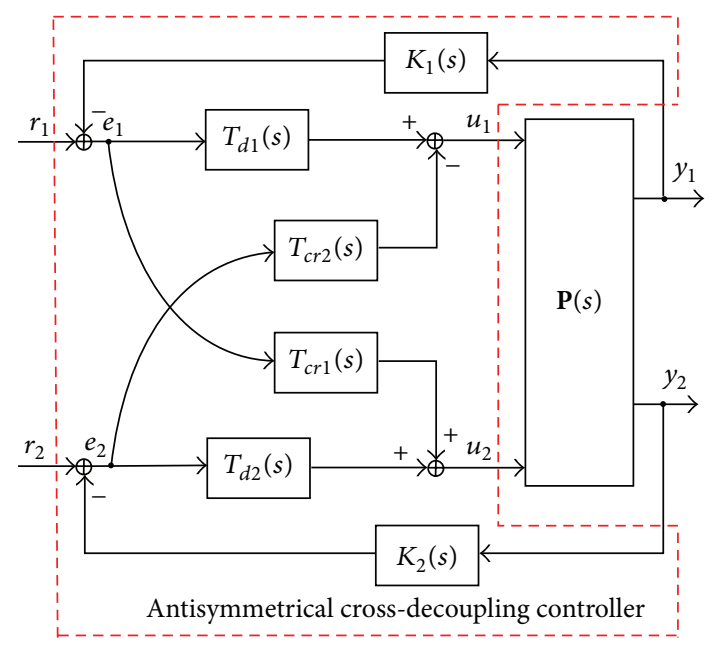

FIGURE 1: Structural scheme of the cross-coupled two-dimensional antisymmetrical system.

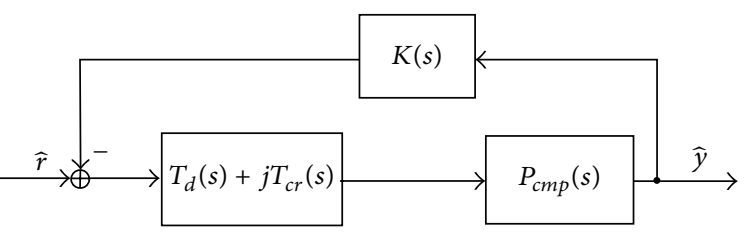

FIGURE 2: Structural scheme of the SISO control system with complex coefficients.

of the cross-coupled antisymmetrical controlled plant can be written as

$$
\left[\begin{array}{cc|cc}
\mathbf{A}_{d} & -\mathbf{A}_{c r} & \mathbf{B} & \mathbf{0} \\
\mathbf{A}_{c r} & \mathbf{A}_{d} & \mathbf{0} & \mathbf{B} \\
\hline \mathbf{C} & \mathbf{0} & \mathbf{0} \\
\mathbf{0} & \mathbf{C} & \mathbf{0}
\end{array}\right],
$$

where $\mathbf{A}_{d}, \mathbf{A}_{c r} \in R^{n \times n}, \mathbf{B} \in R^{n \times 1}$, and $\mathbf{C} \in R^{1 \times n}$.

According to Figure 1, we assume that the state vector of the controlled plant $\mathbf{P}(s)$ is $\left[\begin{array}{ll}\boldsymbol{\alpha} & \boldsymbol{\beta}\end{array}\right]^{T}$, where $\boldsymbol{\alpha}, \boldsymbol{\beta} \in R^{n \times 1}$. The variable reconstruction is performed as follows: $\widehat{r}=r_{1}+j r_{2}$, $\widehat{u}=u_{1}+j u_{2}, \widehat{y}=y_{1}+j y_{2}, \boldsymbol{\varphi}=\boldsymbol{\alpha}+j \boldsymbol{\beta}$. Then, after the variable reconstruction, the original MIMO system with real coefficients is converted into a SISO control system with complex coefficients, which is shown in Figure 2.

Based on the information of the original controlled plant, the system realization of the SISO controlled plant $P_{c m p}(s)$ can be written as

$$
\left[\begin{array}{c|c}
\mathbf{A}_{d}+j \mathbf{A}_{c r} & \mathbf{B} \\
\hline \mathbf{C} & \mathbf{0}
\end{array}\right]
$$

And the open-loop transfer function of the SISO system is given by

$$
G_{c m p}(s)=K(s) P_{c m p}(s)\left(T_{d}(s)+j T_{c r}(s)\right) .
$$




\subsection{Pole Locations Law before and after} Variable Reconstruction

Theorem 1. Suppose that the linear differential equations have the form

$$
D\left[\begin{array}{l}
\boldsymbol{\alpha} \\
\boldsymbol{\beta}
\end{array}\right]=\mathbf{A}\left[\begin{array}{l}
\boldsymbol{\alpha} \\
\boldsymbol{\beta}
\end{array}\right]
$$

where $D$ is the differential operator, $\mathbf{A} \in R^{2 n \times 2 n}$, and $\operatorname{dim}(\boldsymbol{\alpha})=$ $\operatorname{dim}(\boldsymbol{\beta})$. If all the solutions $\left[\begin{array}{ll}\boldsymbol{\alpha} & \boldsymbol{\beta}\end{array}\right]^{T}$ for the above satisfy the following differential equations:

$$
D(\boldsymbol{\alpha}+j \boldsymbol{\beta})=\widetilde{\mathbf{A}}(\boldsymbol{\alpha}+j \boldsymbol{\beta}),
$$

where $\widetilde{\mathbf{A}} \in C^{n \times n}$, then the eigenvalues of the $\mathbf{A}$ and $\widetilde{\mathbf{A}}$ satisfy the following relationships:

(1) If $\widehat{a}+j \widehat{b} \in \sigma_{\lambda}(\widetilde{\mathbf{A}})$, then $\widehat{a} \pm j \widehat{b} \in \sigma_{\lambda}(\mathbf{A})$;

(2) If $a+j b \notin \sigma_{\lambda}(\widetilde{\mathbf{A}})$ and $a-j b \notin \sigma_{\lambda}(\widetilde{\mathbf{A}})$, then $a \pm j b \notin$ $\sigma_{\lambda}(\mathbf{A})$.

Proof. Assume that the basic solution set of $\dot{\mathbf{x}}=\mathbf{A x}$ is $\psi(t)=$ $\left\{\mathbf{v}_{1}(t), \mathbf{v}_{2}(t), \ldots, \mathbf{v}_{n}(t)\right\}$, according to the theory of the linear differential equations, $\mathbf{v}_{i}(t)$ has one of the following three forms: $t^{k} e^{a t} \cos (b t) \mathbf{v}_{c}, t^{k} e^{a t} \sin (b t) \mathbf{v}_{c}$, and $t^{k} e^{a t} \mathbf{v}_{c}$, where $\mathbf{v}_{c}$ is a constant real vector and $\mathbf{v}_{c} \in R^{2 n \times 1}$.

At the same time, $\psi(t)$ can also be given by

$$
\psi(t)=\left\{\left[\begin{array}{l}
\mathbf{v}_{11}(t) \\
\mathbf{v}_{12}(t)
\end{array}\right],\left[\begin{array}{l}
\mathbf{v}_{21}(t) \\
\mathbf{v}_{22}(t)
\end{array}\right], \ldots,\left[\begin{array}{l}
\mathbf{v}_{n 1}(t) \\
\mathbf{v}_{n 2}(t)
\end{array}\right]\right\} .
$$

As for the complex-coefficient differential equations $\dot{\mathbf{x}}=$ $\widetilde{\mathbf{A}} \mathbf{x}$, its basic solution set can be described as $\widetilde{\psi}(t)=$ $\left\{\widetilde{\mathbf{v}}_{1}(t), \widetilde{\mathbf{v}}_{2}(t), \ldots, \widetilde{\mathbf{v}}_{m}(t)\right\}$, where $\widetilde{\mathbf{v}}_{i}(t)$ has a form of $t^{k} e^{(\hat{a}+j \hat{b}) t} \widehat{\mathbf{v}}_{c}$, where $\widehat{\mathbf{v}}_{c}$ is the constant complex vector and $\widehat{\mathbf{v}}_{c} \in C^{n \times 1}$.

Define the following function space:

$$
\begin{aligned}
& \boldsymbol{v}_{r}=\operatorname{span}\left\{\left[\begin{array}{l}
\mathbf{v}_{11}(t) \\
\mathbf{v}_{12}(t)
\end{array}\right],\left[\begin{array}{c}
\mathbf{v}_{21}(t) \\
\mathbf{v}_{22}(t)
\end{array}\right], \ldots,\left[\begin{array}{c}
\mathbf{v}_{n 1}(t) \\
\mathbf{v}_{n 2}(t)
\end{array}\right]\right\}, \\
& \boldsymbol{v}_{r c}=\operatorname{span}\left\{\mathbf{v}_{11}(t)+j \mathbf{v}_{12}(t), \mathbf{v}_{21}(t)\right. \\
& \left.+j \mathbf{v}_{22}(t), \ldots, \mathbf{v}_{n 1}(t)+j \mathbf{v}_{n 2}(t)\right\}, \\
& \boldsymbol{v}_{c}=\operatorname{span}\left\{\widetilde{\mathbf{v}}_{1}(t), \widetilde{\mathbf{v}}_{2}(t), \ldots, \widetilde{\mathbf{v}}_{m}(t)\right\} .
\end{aligned}
$$

From the proposition, it can be obtained that $\boldsymbol{v}_{r c} \subset \boldsymbol{v}_{c}$. According to the property of the general solution for the ordinary differential equations, one can obtain that $\operatorname{dim}\left(\boldsymbol{v}_{c}\right)=$ $n=\operatorname{dim}\left(\boldsymbol{v}_{r c}\right)$. Accordingly, $\boldsymbol{v}_{r c}=\boldsymbol{v}_{c}$.

As for any $\widehat{\mathbf{v}}, \widetilde{\mathbf{v}} \in C^{m \times 1}, \widehat{\mathbf{v}}, \widetilde{\mathbf{v}} \neq \overrightarrow{0}$, it can be proven that $e^{(\hat{a}+j \hat{b}) t} \widetilde{\mathbf{v}}, e^{a t} \cos (b t) \widehat{\mathbf{v}}$, and $e^{b t} \sin (b t) \widehat{\mathbf{v}}$ are linearly independent if $\widehat{a}+j \widehat{b} \neq a+j b$. When $\widehat{a}+j \widehat{b} \in \sigma_{\lambda}(\widetilde{\mathbf{A}}), \widetilde{\mathbf{v}}$ is the corresponding eigenvector; according to the format of the solution to the ordinary differential equations, $e^{(\hat{a}+j \widehat{b}) t} \widetilde{\mathbf{v}}$ is the solution of (5); that is, $e^{(\widehat{a}+j \widehat{b}) t} \widetilde{\mathbf{v}} \in \boldsymbol{v}_{c}=\boldsymbol{v}_{r c}$. When $\widehat{a}+j \widehat{b} \in$ $\sigma_{\lambda}(\widetilde{\mathbf{A}}), \widetilde{\mathbf{v}}$ is the corresponding eigenvector, according to the format of the solution to the ordinary differential equations, $e^{(\hat{a}+j \hat{b}) t} \widetilde{\mathbf{v}}$ is the solution to (5), that is, $e^{(\widehat{a}+j \hat{b}) t} \widetilde{\mathbf{v}} \in \boldsymbol{v}_{c}=\boldsymbol{v}_{r c}$. If $\widehat{a}+\hat{j b} \notin \sigma_{\lambda}(\mathbf{A})$, from the format of the fundamentalsolution matrix for (4), it can be derived that $e^{(\widehat{a}+j \hat{b}) t} \widetilde{\mathbf{v}} \notin \boldsymbol{v}_{r c}$ since any $\mathbf{v}_{i 1}(t)+j \mathbf{v}_{i 2}(t)$ and $e^{(\widehat{a}+j \widehat{b}) t} \widetilde{\mathbf{v}}$ are linearly independent and $\left\{\mathbf{v}_{i 1}(t)+j \mathbf{v}_{i 2}(t)\right\}$ is the set of the base vectors of $\boldsymbol{v}_{r c}$. And this contradicts the fact that $e^{(\widehat{a}+j \widehat{b}) t} \widetilde{\mathbf{v}} \in \boldsymbol{v}_{r c}$. Accordingly, proposition (1) is right.

Simultaneously, if $a \pm j b \in \sigma_{\lambda}(\mathbf{A})$, then both $e^{a t} \cos (b t) \mathbf{v}$ and $\mathbf{v} \in R^{2 n \times 1}$ are the solutions for (4). One can define $\mathbf{v}=\left\{\begin{array}{l}\mathbf{v}_{1} \\ \mathbf{v}_{2}\end{array}\right\}$, where $\mathbf{v}_{1}, \mathbf{v}_{2} \in R^{n \times 1}$; then one can obtain that $e^{a t} \cos (b t)\left(\mathbf{v}_{1}+j \mathbf{v}_{2}\right) \in \operatorname{span}\left\{\widetilde{\mathbf{v}}_{1}(t), \widetilde{\mathbf{v}}_{2}(t), \ldots, \widetilde{\mathbf{v}}_{n}(t)\right\}$. If $a+$ $j b \notin \sigma_{\lambda}(\widetilde{\mathbf{A}})$ and $a-j b \notin \sigma_{\lambda}(\widetilde{\mathbf{A}})$, then it can be drawn that $e^{a t} \cos (b t)\left(\mathbf{v}_{1}+j \mathbf{v}_{2}\right) \notin \operatorname{span}\left\{\widetilde{\mathbf{v}}_{1}(t), \widetilde{\mathbf{v}}_{2}(t), \ldots, \widetilde{\mathbf{v}}_{n}(t)\right\}$, which is not in accordance with $e^{a t} \cos (b t)\left(\mathbf{v}_{1}+j \mathbf{v}_{2}\right) \in$ $\operatorname{span}\left\{\widetilde{\mathbf{v}}_{1}(t), \widetilde{\mathbf{v}}_{2}(t), \ldots, \widetilde{\mathbf{v}}_{n}(t)\right\}$. Accordingly, proposition (2) is positive. This completes the proof.

Theorem 2. The systems before and after variable reconstruction satisfy the pole locations law presented in Theorem 1.

Proof. Note that system matrix determines the pole locations; therefore, it should be obtained at first. Since $T_{d 1}(s)=T_{d 2}(s)$, their system realization is given by

$$
\left[\begin{array}{c|c}
\widehat{\mathbf{A}}_{d} & \widehat{\mathbf{B}}_{d} \\
\hline \widehat{\mathbf{C}}_{d} & \widehat{\mathbf{D}}_{d}
\end{array}\right] .
$$

And the state variables of $T_{d 1}(s)$ and $T_{d 2}(s)$ are $\mathbf{x}_{d 1}, \mathbf{x}_{d 2}$, respectively.

Similarly the system realization of $T_{c r 1}(s)$ and $T_{c r 2}(s)$ is given by

$$
\left[\begin{array}{c|c}
\widehat{\mathbf{A}}_{c r} & \widehat{\mathbf{B}}_{c r} \\
\hline \widehat{\mathbf{C}}_{c r} & \widehat{\mathbf{D}}_{c r}
\end{array}\right]
$$

And the state variables of $T_{c r 1}(s)$ and $T_{c r 2}(s)$ are, respectively, $\mathbf{x}_{c r 1}$ and $\mathbf{x}_{c r 2}$.

Also, the system realization of $K_{1}(s)$ and $K_{2}(s)$ can be described as

$$
\left[\begin{array}{c|c}
\widehat{\mathbf{A}}_{k} & \widehat{\mathbf{B}}_{k} \\
\hline \widehat{\mathbf{C}}_{k} & \widehat{\mathbf{D}}_{k}
\end{array}\right],
$$

with the state variables of $\mathbf{x}_{k 1}$ and $\mathbf{x}_{k 2}$, respectively.

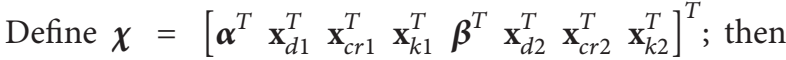
$\dot{\chi}=\mathbf{A} \boldsymbol{\chi}$. According to the closed-loop structure diagram of the control system, one has

$$
\begin{aligned}
\dot{\boldsymbol{\alpha}}= & \left(\mathbf{A}_{d}-\mathbf{B}_{\mathbf{D}_{d}} \widehat{\mathbf{D}}_{k} \mathbf{C}\right) \boldsymbol{\alpha}+\mathbf{B} \widehat{\mathbf{C}}_{d} \mathbf{x}_{d 1} \\
& -\mathbf{B} \widehat{\mathbf{D}}_{d} \widehat{\mathbf{C}}_{k} \mathbf{x}_{k 1}-\left(\mathbf{A}_{c r}-\mathbf{B} \widehat{\mathbf{D}}_{c r} \widehat{\mathbf{D}}_{k} \mathbf{C}\right) \boldsymbol{\beta} \\
& -\mathbf{B} \widehat{\mathbf{C}}_{c r} \mathbf{x}_{c r 2}+\mathbf{B} \widehat{\mathbf{D}}_{c r} \widehat{\mathbf{C}}_{k} \mathbf{x}_{k 2}, \\
\dot{\boldsymbol{\beta}}=( & \left.\mathbf{A}_{c r}-\mathbf{B} \widehat{\mathbf{D}}_{c r} \widehat{\mathbf{D}}_{k} \mathbf{C}\right) \boldsymbol{\alpha}+\mathbf{B} \widehat{\mathbf{C}}_{c r} \mathbf{x}_{c r 1} \\
& -\mathbf{B} \widehat{\mathbf{D}}_{c r} \widehat{\mathbf{C}}_{k} \mathbf{x}_{k 1}+\left(\mathbf{A}_{d}-\mathbf{B} \widehat{\mathbf{D}}_{d} \widehat{\mathbf{D}}_{k} \mathbf{C}\right) \boldsymbol{\beta} \\
& +\mathbf{B} \widehat{\mathbf{C}}_{d} \mathbf{x}_{d 2}-\mathbf{B} \widehat{\mathbf{D}}_{d} \widehat{\mathbf{C}}_{k} \mathbf{x}_{k 2} .
\end{aligned}
$$


Then the system matrix of the whole system before variable reconstruction is given by

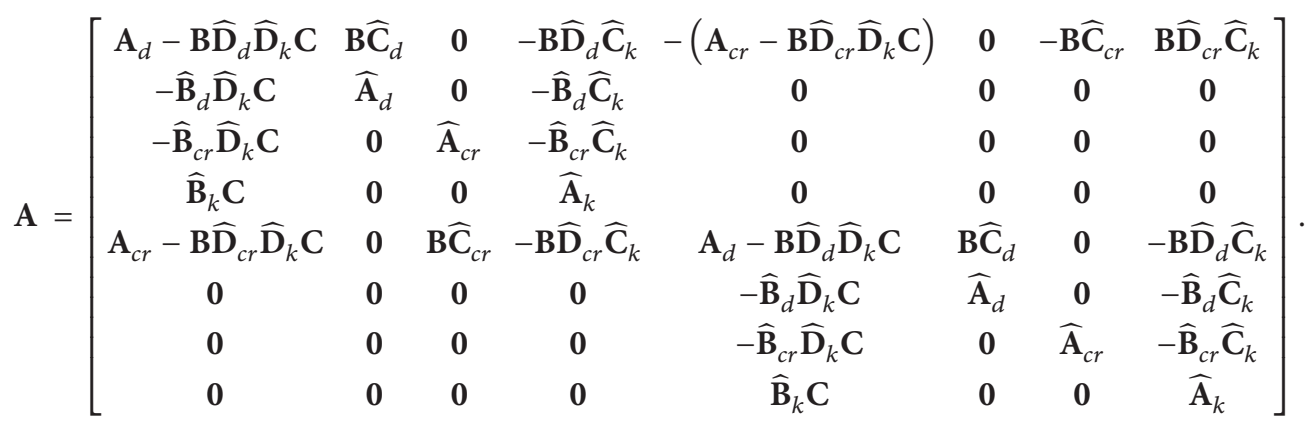

Define the new state variables $\widetilde{\chi}=$ according to (12) and Theorem 1 , the corresponding system $\left[\begin{array}{llll}\boldsymbol{\alpha}^{T}+j \boldsymbol{\beta}^{T} & \mathbf{x}_{d 1}^{T}+j \mathbf{x}_{d 2}^{T} \quad \mathbf{x}_{c r 1}^{T}+j \mathbf{x}_{c r 2}^{T} \quad \mathbf{x}_{k 1}^{T}+j \mathbf{x}_{k 2}^{T}\end{array}\right]^{T} ; \quad$ then, $\quad$ matrix $\tilde{\mathbf{A}}$ of $\tilde{\boldsymbol{\chi}}$ can be derived as

$$
\widetilde{\mathbf{A}}=\left[\begin{array}{cccc}
\left(\mathbf{A}_{d}-\mathbf{B} \widehat{\mathbf{D}}_{c r} \widehat{\mathbf{D}}_{k} \mathbf{C}\right)+j\left(\mathbf{A}_{c r}-\mathbf{B} \widehat{\mathbf{D}}_{c r} \widehat{\mathbf{D}}_{k} \mathbf{C}\right) & \mathbf{B} \widehat{\mathbf{C}}_{d} & j \mathbf{B} \widehat{\mathbf{C}}_{c r} & -\mathbf{B}\left(\widehat{\mathbf{D}}_{d}+j \widehat{\mathbf{D}}_{c r}\right) \widehat{\mathbf{C}}_{k} \\
-\widehat{\mathbf{B}}_{d} \widehat{\mathbf{D}}_{k} \mathbf{C} & \widehat{\mathbf{A}}_{d} & \mathbf{0} & -\widehat{\mathbf{B}}_{d} \widehat{\mathbf{C}}_{k} \\
-\widehat{\mathbf{B}}_{c r} \widehat{\mathbf{D}}_{k} \mathbf{C} & \mathbf{0} & \widehat{\mathbf{A}}_{c r} & -\widehat{\mathbf{B}}_{c r} \widehat{\mathbf{C}}_{k} \\
\widehat{\mathbf{B}}_{k} \mathbf{C} & \mathbf{0} & \mathbf{0} & \widehat{\mathbf{A}}_{k}
\end{array}\right] .
$$

As for the SISO system with complex coefficients, the system realization of $T_{d}(s)+j T_{c r}(s)$ can be derived from the system realizations of $T_{d}(s)$ and $T_{c r}(s)$ as follows:

$$
\left[\begin{array}{cc|c}
\widehat{\mathbf{A}}_{d} & \mathbf{0} & \widehat{\mathbf{B}}_{d} \\
\mathbf{0} & \widehat{\mathbf{A}}_{c r} & \widehat{\mathbf{B}}_{c r} \\
\hline \widehat{\mathbf{C}}_{d} & j \widehat{\mathbf{C}}_{c r} & \widehat{\mathbf{D}}_{d}+j \widehat{\mathbf{D}}_{c r}
\end{array}\right]
$$

Similarly, the complex-coefficient system matrix of the SISO system after variable reconstruction can be derived as

$$
\mathbf{A}_{c m p}=\left[\begin{array}{cccc}
\left(\mathbf{A}_{d}-\mathbf{B} \widehat{\mathbf{D}}_{c r} \widehat{\mathbf{D}}_{k} \mathbf{C}\right)+j\left(\mathbf{A}_{c r}-\mathbf{B} \widehat{\mathbf{D}}_{c r} \widehat{\mathbf{D}}_{k} \mathbf{C}\right) & \mathbf{B} \widehat{\mathbf{C}}_{d} & j \mathbf{B} \widehat{\mathbf{C}}_{c r} & -\mathbf{B}\left(\widehat{\mathbf{D}}_{d}+j \widehat{\mathbf{D}}_{c r}\right) \widehat{\mathbf{C}}_{k} \\
-\widehat{\mathbf{B}}_{d} \widehat{\mathbf{D}}_{k} \mathbf{C} & \widehat{\mathbf{A}}_{d} & \mathbf{0} & \mathbf{0} \\
-\widehat{\mathbf{B}}_{c} \widehat{\mathbf{D}}_{k} \mathbf{C} & \mathbf{0} & \widehat{\mathbf{A}}_{c r} & \mathbf{0} \\
\widehat{\mathbf{B}}_{k} \mathbf{C} & \mathbf{0} & \mathbf{0} & \widehat{\mathbf{A}}_{k}
\end{array}\right]
$$

From (13) and (15), it can be drawn that $\mathbf{A}_{c m p}=\widehat{\mathbf{A}}$. Accordingly, the original system and the new system are in line with the same pole locations laws with that of Theorem 1 . This concludes the proof.

\subsection{Controllability and Observability before and after Variable Reconstruction}

Lemma 3. If $(\mathbf{A}+j \mathbf{B})^{n}=\mathbf{A}_{n}+j \mathbf{B}_{n}$, then $\left[\begin{array}{cc}\mathbf{A} & \mathbf{B} \\ -\mathbf{B} & \mathbf{A}\end{array}\right]^{n}=\left[\begin{array}{cc}\mathbf{A}_{n} & \mathbf{B}_{n} \\ -\mathbf{B}_{n} & \mathbf{A}_{n}\end{array}\right]$, where $\mathbf{A}, \mathbf{B}, \mathbf{A}_{n}, \mathbf{B}_{n} \in R^{m \times m}$.

Proof. When $n=1$, the conclusion holds obviously. Suppose that the conclusion also holds when $n=N$; then when
$n=N+1,(\mathbf{A}+j \mathbf{B})^{n+1}=\left(\mathbf{A}_{n}+j \mathbf{B}_{n}\right)(\mathbf{A}+j \mathbf{B})=$ $\left(\mathbf{A}_{n} \mathbf{A}-\mathbf{B}_{n} \mathbf{B}\right)+j\left(\mathbf{B}_{n} \mathbf{A}+\mathbf{A}_{n} \mathbf{B}\right)$; that is, $\mathbf{A}_{n+1}=\mathbf{A}_{n} \mathbf{A}-\mathbf{B}_{n} \mathbf{B}$ and $\mathbf{B}_{n+1}=\mathbf{B}_{n} \mathbf{A}+\mathbf{A}_{n} \mathbf{B}$.

Similarly, $\left[\begin{array}{cc}\mathbf{A} & \mathbf{B} \\ -\mathbf{B} & \mathbf{A}\end{array}\right]^{n+1}=\left[\begin{array}{cc}\mathbf{A}_{n} & \mathbf{B}_{n} \\ -\mathbf{B}_{n} & \mathbf{A}_{n}\end{array}\right]\left[\begin{array}{cc}\mathbf{A} & \mathbf{B} \\ -\mathbf{B} & \mathbf{A}\end{array}\right]=$ $\left[\begin{array}{cc}\mathbf{A}_{n} \mathbf{A}-\mathbf{B}_{n} \mathbf{B} & \mathbf{A}_{n} \mathbf{B}+\mathbf{B}_{n} \mathbf{A} \\ -\left(\mathbf{A}_{n} \mathbf{B}+\mathbf{B}_{n} \mathbf{A}\right) & \mathbf{A}_{n} \mathbf{A}-\mathbf{B}_{n} \mathbf{B}\end{array}\right]=\left[\begin{array}{cc}\mathbf{A}_{n+1} & \mathbf{B}_{n+1} \\ -\mathbf{B}_{n+1} & \mathbf{A}_{n+1}\end{array}\right]$. This concludes the proof.

Theorem 4. If the SISO system (2) is controllable and observable, that is, $\operatorname{rank}\left(\mathbf{B} \widetilde{\mathbf{A}} \mathbf{B} \cdots \widetilde{\mathbf{A}}^{n-1} \mathbf{B}\right)=n$ and $\operatorname{rank}\left(\left[\begin{array}{lllll}\mathbf{C} & \mathbf{C} \widetilde{\mathbf{A}} & \cdots & \mathbf{C} \widetilde{\mathbf{A}}^{n-1}\end{array}\right]^{T}\right)=n$, where $\widetilde{\mathbf{A}}=\mathbf{A}_{d}+j \mathbf{A}_{c r}$, then the MIMO system (1) is controllable and observable too. 
Proof. Denote $\left[\begin{array}{cc}\mathbf{A}_{d} & -\mathbf{A}_{c r} \\ \mathbf{A}_{c r} & \mathbf{A}_{d}\end{array}\right]^{n}=\left[\begin{array}{cc}\mathbf{A}_{d n} & -\mathbf{A}_{c r n} \\ \mathbf{A}_{c r n} & \mathbf{A}_{d n}\end{array}\right]$, where $n \in \mathbb{N}$. If the MIMO system (1) is not controllable, then

$$
\begin{aligned}
\operatorname{rank} & {\left[\begin{array}{ccccccc}
\mathbf{A}_{d 0} \mathbf{B} & -\mathbf{A}_{c r 0} \mathbf{B} & \mathbf{A}_{d 1} \mathbf{B} & -\mathbf{A}_{c r 1} \mathbf{B} & \cdots & \mathbf{A}_{d(n-1)} \mathbf{B} & -\mathbf{A}_{c r(n-1)} \mathbf{B} \\
\mathbf{A}_{c r 0} \mathbf{B} & \mathbf{A}_{d 0} \mathbf{B} & \mathbf{A}_{c r 1} \mathbf{B} & \mathbf{A}_{d 1} \mathbf{B} & \cdots & \mathbf{A}_{c r(n-1)} \mathbf{B} & \mathbf{A}_{d(n-1)} \mathbf{B}
\end{array}\right] } \\
& <2 n .
\end{aligned}
$$

That is, there exists a set of real numbers $\left\{\gamma_{0}, \gamma_{1}, \ldots, \gamma_{n-1}\right.$, $\left.\lambda_{0}, \lambda_{1}, \ldots, \lambda_{n-1}\right\}$ which are not all zeros so that

$$
\begin{aligned}
& \sum_{i=0}^{n-1} \gamma_{i} \mathbf{A}_{d i} \mathbf{B}-\sum_{i=0}^{n-1} \lambda_{i} \mathbf{A}_{c r i} \mathbf{B}=0, \\
& \sum_{i=0}^{n-1} \gamma_{i} \mathbf{A}_{d i} \mathbf{B}+\sum_{i=0}^{n-1} \lambda_{i} \mathbf{A}_{c r i} \mathbf{B}=0 .
\end{aligned}
$$

According to Lemma 3, it can be obtained that $\widetilde{\mathbf{A}}^{i}=\mathbf{A}_{d i}+$ $j \mathbf{A}_{c r i}$. Suppose $\xi_{i}=\gamma_{i}+j \lambda_{i}$; then, from (17), it can be drawn that $\sum_{i=0}^{n-1} \xi_{i} \widetilde{\mathbf{A}}^{i} \mathbf{B}=0$, which is not in accordance with the assumption.

Similarly, it can be proven that if the SISO system (2) is observable, then the MIMO system (1) is observable too. Proof of the proposition is completed.

\subsection{Complex-Coefficient Frequency Domain Stability Crite-} rion. According to Theorems 1-4, the following corollary can be achieved.

Corollary 5. As for the complex-coefficient system (15), the sufficient and necessary condition for the system stability is that all the characteristic roots lie in the left half plane (LHP). And the sufficient and necessary condition for the system critical stability is that there is at least a pure imaginary root and there is no right-half plane (RHP) pole.

From the analysis above, it can be drawn that the stability of the cross-coupled antisymmetrical system can be demonstrated by that of the corresponding complex-coefficient system. Note that the argument principle applies to the complex variable function which is defined on the complex plane, and the complex-coefficient open-loop transfer function $G_{c m p}$ also belongs to this class of complex variable functions. Since the Nyquist stability criterion is based on the argument principle and the system controllability and observability are the precondition of Nyquist stability criterion, the corresponding SISO system can be employed to analyze the stability of the original control system by using complex-coefficient Nyquist stability criterion. Accordingly, one can achieve the following stability analysis method based on complex-coefficient frequency characteristics. Denote $\operatorname{enc}_{D}\left(G_{c m p}(s),-1+j 0\right)$ as the number of anticlockwise encirclements of the $-1+j 0$ point by the $G_{c m p}(s)$ locus as a representative point $s$ traces on the path $D$ in the clockwise direction, where $D$ is the semicircle contour that encloses the entire right-half $s$ plane. Then one can obtain the following stability criterion for twodimensional cross-coupled antisymmetrical systems.

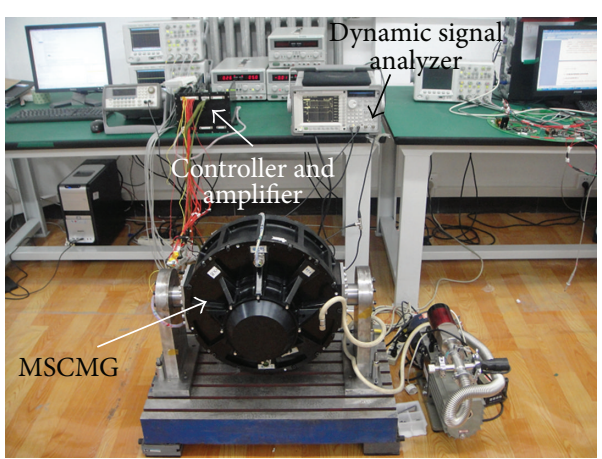

FIGURE 3: Photograph of the experimental setup.

Criterion 1. As for the cross-coupled two-dimensional antisymmetrical system (12), if its corresponding SISO controlled plant (2) is both controllable and observable, and its number of the RHP poles is $N_{O R}$, then the sufficient and necessary condition for the stability of system (12) is that $\operatorname{enc}_{D}\left(G_{c m p}(s),-1+j 0\right)=N_{O R}$.

Compared with the traditional Nyquist stability criterion for the multi variable systems, the proposed one greatly simplified the stability analysis since the complex multivariable system has become a single-variable system.

In fact, as for a special controlled plant, this criterion can be further developed according to its practical physical meaning, for example, the MSR system with heavy gyroscopic effects; it can be proven that the closed-loop righthalf-poles and left-half-poles of the complex-coefficient SISO system denote the forward and backward whirling modes of the MSR respectively, which is the theoretical base for the whirling modes stability criterion [41].

\section{Simulation and Experimental Results}

Note that the large magnetically suspended control moment gyroscope (MSCMG) has a rigid MSR with significant crosscoupling effects which is a typical coupled-coupled antisymmetrical system; thus, it is taken as the example. To demonstrate the effectiveness and superiority of the proposed stability criterion, comparative simulation between the proposed method and the traditional one (root locus with respect to rotor speed) and experiments have been performed.

3.1. Experiment Setup. Figure 3 shows the experimental setup, where the MSR has two 2-degree-of-freedom (DOF) radial $\mathrm{MBs}$ and two single-DOF axial MBs, which are driven by five $\mathrm{H}$-bridge unipolar switching power amplifiers independently [42], realizing 5-DOF active control.

The well-known decentralized PID plus filtered cross feedback control approach [34] is adopted. The main parameters of the MSCMG and the controller coefficients used in simulation and experiments have been listed in Tables 1 and 2.

The experiments are performed in a TMS320C32 digital signal processor (DSP) board. The total digital control system 
TABLE 1: System parameters of the MSCMG.

\begin{tabular}{lc}
\hline Parameter & Value \\
\hline$m$ & $56 \mathrm{~kg}$ \\
$J_{x}$ & $0.6032 \mathrm{~kg} \cdot \mathrm{m}^{2}$ \\
$J_{z}$ & $0.7958 \mathrm{~kg} \cdot \mathrm{m}^{2}$ \\
$k_{i}$ & $1140 \mathrm{~N} / \mathrm{A}$ \\
$k_{s}$ & 75926 \\
$x_{a}$ & $120 \mu \mathrm{m}$ \\
$J_{y}$ & $0.6032 \mathrm{~kg} \cdot \mathrm{m}^{2}$ \\
$l_{s}$ & $0.177 \mathrm{~m}$ \\
$k_{h}$ & $2.83 \mathrm{~N} / \mu \mathrm{m}$ \\
$l_{m}$ & $0.113 \mathrm{~m}$ \\
\hline
\end{tabular}

TABle 2: Parameters of the controller.

\begin{tabular}{lc}
\hline Parameter & Value \\
\hline$k_{\mathrm{am}}$ & 2.0 \\
$k_{p}$ & 0.167 \\
$k_{d}$ & 0.0008 \\
$k_{l c}$ & 0.00015 \\
$f_{h}$ & $280 \mathrm{~Hz}$ \\
$i_{c}$ & 10.0 \\
$k_{i}$ & 1.7 \\
$k_{h c}$ & 0.0045 \\
$k_{c}$ & 1.0 \\
$f_{l}$ & $30 \mathrm{~Hz}$ \\
\hline
\end{tabular}

delay can be tested, which is about $150 \mu \mathrm{s}$. To facilitate drawing the root locus diagrams [43] and compare fairly, the delay unit is simplified as its second-order Taylor's series approximation when drawing the root locus and the complexcoefficient frequency characteristics plots; that is,

$$
g_{\tau}(s)=e^{-\tau s} \approx \frac{1}{1+\tau s+0.5 \tau^{2} s^{2}}
$$

where $\tau=0.00017$. In fact, this approximate has little influence on the stability analysis for the MSR system since the interested critical stability frequencies of the whirling modes are relatively low. That is, the phase errors at the critical stability frequencies are tolerant.

The closed-loop rotation dynamic model with the antisymmetrical cross-decoupling controller can be given by [41, $44]$

$$
\begin{aligned}
& J_{r} \ddot{\beta}(t)-J_{z} \Omega \dot{\alpha}(t)-2 k_{h} l_{m}^{2} \beta(t) \\
& \quad=-2 l_{m} l_{s} k_{i} k_{s} g_{a} g_{\tau} g_{f}\left[g_{b}(\beta(t))+g_{c r}(\alpha(t))\right], \\
& J_{r} \ddot{\alpha}(t)+J_{z} \Omega \dot{\beta}(t)-2 k_{h} l_{m}^{2} \alpha(t) \\
& \quad=-2 l_{m} l_{s} k_{i} k_{s} g_{a} g_{\tau} g_{f}\left[g_{b}(\alpha(t))-g_{c r}(\beta(t))\right],
\end{aligned}
$$

where $J_{r}$ and $J_{z}$ are the momenta of inertia of the rotor about the radial and axial directions, respectively; $\Omega$ is the rotor speed; $l_{m}$ and $l_{s}$ are the distances from the geometry center of the MSR to the radial and the radial sensor; $\alpha$ and $\beta$ are the rotor angular displacements about the $X$ - and $Y$ axes; $g_{b}, g_{c r}, g_{a}, g_{f}$, and $g_{\tau}$ denote the basic controller, the antisymmetrical cross-decoupling controller, the switching power amplifier, the antialias filter, and the control system delay unit, respectively; $k_{h}$ is the force-displacement factor of the radial magnetic bearings, and $k_{s}$ is the proportional gain of the displacement sensor.

The open-loop transfer function of the SISO system with complex coefficients can be derived as [44]

$$
G_{c m p}(s)=\frac{2 l_{m} l_{s} k_{i} k_{s} g_{a}(s) g_{\tau}(s) g_{f}\left(g_{b}(s)+j g_{c r}(s)\right)}{J_{r} s^{2}-j J_{z} \Omega s-2 k_{h} l_{m}^{2}} .
$$

According to the definition used by logarithmic frequency stability criterion [45], we make the following definitions: $N_{p+}, N_{p-}$ denote the number of the positive and negative phase cross-over points of $G_{c m p}(j \omega)$ in the set of $L_{P}=\left\{\omega|\omega \geq 0, \log | G_{c m p}(j \omega) \mid \geq 0\right\}$, when $\omega$ changes from 0 to $+\infty ; N_{s f+}, N_{s f-}$ denote the number of the positive and negative phase cross-over points of $G_{c m p}(j \omega)$ in $L_{S}=\{\sigma \mid \sigma \geq$ $\left.0, \log \left|G_{c m p}(\sigma)\right| \geq 0\right\}$, when $\sigma$ decreases from $+\infty$ to $0 ; N_{s b+}$, $N_{s b-}$ are the number of the positive and negative phase crossover points of $G_{c m p}(\sigma)$ in $L_{s}$, when $\sigma$ increases from 0 to $+\infty$; and $N_{n+}, N_{n-}$ denote the number of the positive and negative phase cross-over points of $G_{c m p}(j \omega)$ in $L_{N}=\{\omega \mid \omega \leq 0$, $\left.\log \left|G_{c m p}(s)\right| \geq 0\right\}$ when $\omega$ changes from $-\infty$ to 0 . According to the proposed Criterion 1 and the whirling modes stability criterion for a MSR with bending modes [41], one can further obtain the following whirling modes stability criterion for the rigid MSR without bending modes.

Criterion 2. As for the rigid MSR system (19), the sufficient and necessary conditions for the nutation and precession modes stability are $N_{\mathrm{OFR}}=N_{p+}+N_{s f+}-N_{p_{-}}-N_{s f-}$ and $N_{\mathrm{OBR}}=N_{n+}+N_{s b+}-N_{n-}-N_{s b-}$, respectively.

3.2. Simulation and Experimental Results. At first, under the conditions that the cross proportion is $48 \mathrm{~Hz}$, the Bode diagram is drawn, respectively, at the rotation speed of $140 \mathrm{~Hz}$ and $145 \mathrm{~Hz}$, which is shown in Figure 4. Substituting the system parameters and rotor speed into (20), it can be calculated that $N_{O F R}=0$ and $N_{O B R}=0$. From Figure 4(a), it can be achieved that $N_{p+}+N_{s f+}-N_{p-}-N_{s f-}=0+0.5-0.5-0=$ 0 and $N_{n+}+N_{s b+}-N_{n-}-N_{s b-}=1+0-0.5-0.5=0$. According to the whirling modes stability criterion, $N_{O F R}=$ $N_{p+}+N_{s f+}-N_{p_{-}}-N_{s f-}, N_{\mathrm{OBR}}=N_{n+}+N_{s b+}-N_{n-}-N_{s b-}$; that is, both the forward and backward whirling modes are stable. Simultaneously, from Figure 4(b), it can be achieved that $N_{p+}+N_{s f+}-N_{p-}-N_{s f-}=0+0.5-1.5-0=-1$ and $N_{n+}+N_{s b+}-$ $N_{n-}-N_{s b-}=1+0-0.5-0.5=0$. Therefore, the backward whirling mode is stable, while the forward whirling mode is unstable at the speed of $145 \mathrm{~Hz}$. The root locus with respect to the rotor speed is drawn, which is shown in Figure 5. The speed range is from $0 \mathrm{~Hz}$ to $150 \mathrm{~Hz}$ and it is plotted every $5 \mathrm{~Hz}$. According to Figure 5, it can be derived that the system is stable at the speed of $140 \mathrm{~Hz}$ while unstable at the speed of $145 \mathrm{~Hz}$; that is, the margin speed is located between $140 \mathrm{~Hz}$ and $145 \mathrm{~Hz}$, which is in accordance with the conclusion drawn 


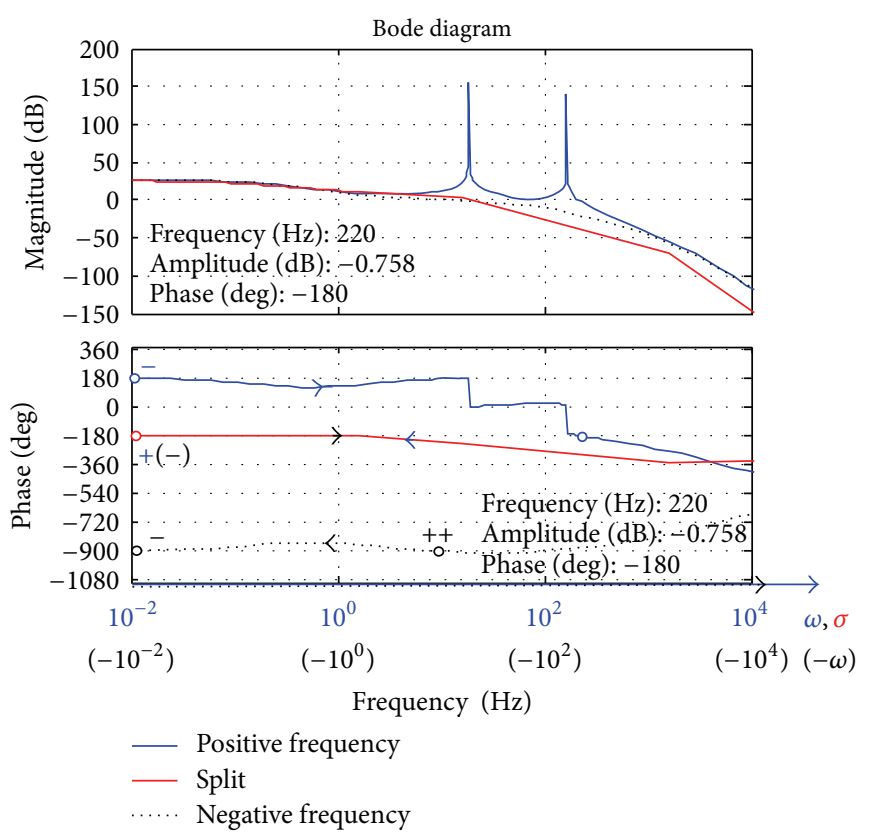

(a)

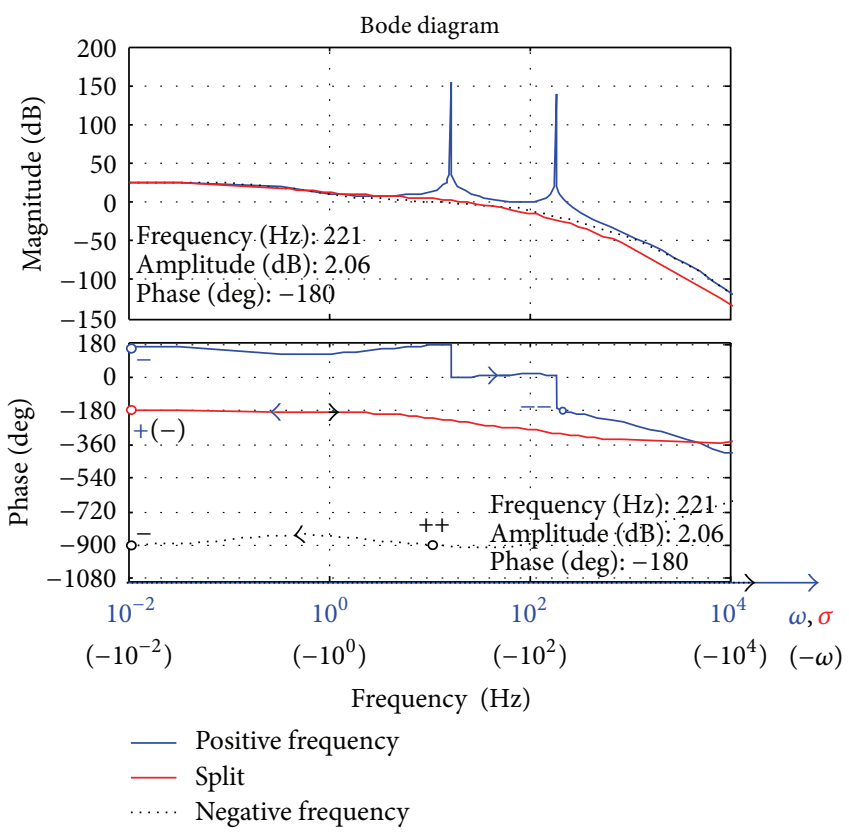

(b)

Figure 4: Complex-coefficient frequency characteristics of the SISO system with $48 \mathrm{~Hz}$ cross proportion and different rotation speeds. (a) 140 Hz. (b) $145 \mathrm{~Hz}$.

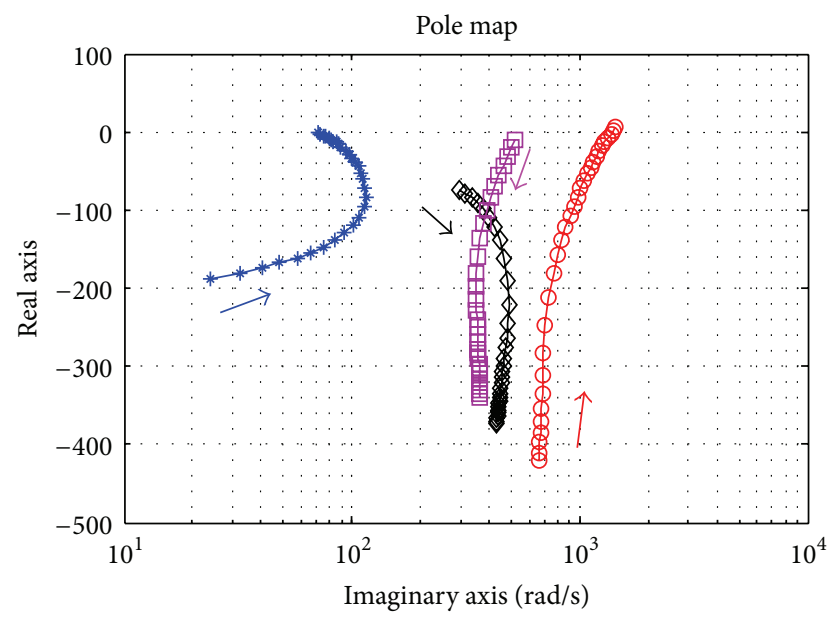

FIGURE 5: Root locus with respect to rotor speed from $0 \mathrm{~Hz}$ to $150 \mathrm{~Hz}$. Note: the arrowhead denotes the increased direction of rotor speed from 0 to $150 \mathrm{~Hz}$.

from the proposed stability criterion. It is necessary to note that phase margin of the nutation and precession modes can be obtained from the proposed stability criterion, while it cannot be obtained from the root locus.

Then we set the cross proportion to be $150 \mathrm{~Hz}$, the corresponding simulation and experiments have been developed too, and their results are shown in Figures 6 and 7. Also, it can be obtained that $N_{\text {OFR }}=0$ and $N_{O B R}=0$ at the speeds of $200 \mathrm{~Hz}$ and $205 \mathrm{~Hz}$ and $N_{\text {OFR }}=1$ and $N_{\text {OBR }}=0$ at the speeds of $55 \mathrm{~Hz}$ and $50 \mathrm{~Hz}$. From Figure 6(a), one can obtain that $N_{p+}+N_{s f+^{+}}-N_{p_{-}}-N_{s f-}=0+0.5-0.5-0=0$,
$N_{n+}+N_{s b+}-N_{n-}-N_{s b-}=1+0-0.5-0.5=0$. According to the proposed criterion, it can be drawn that both the forward and backward whirling modes are stable at the speed of $200 \mathrm{~Hz}$. From Figure 6(b), one can obtain that $N_{\mathrm{OFR}} \neq N_{p+}+$ $N_{s f+}-N_{p-}-N_{s f-}$ and $N_{\mathrm{OBR}}=N_{n+}+N_{s b+}-N_{n-}-N_{s b-}$; that is, the backward whirling mode remains stable while the forward whirling mode loses its stability at the speed of $205 \mathrm{~Hz}$. Similarly, from Figures 6(c) and 6(d), the conclusion can be achieved that both the forward and backward modes are stable at the speed of $55 \mathrm{~Hz}$ while the backward whirling mode is unstable at the speed of $50 \mathrm{~Hz}$. Also, from Figures 6(c) and 6(d), it can be calculated that the phase margins of the forward whirling mode at the speed of $50 \mathrm{~Hz}$ and $55 \mathrm{~Hz}$ are 56 degree and 52 degrees, respectively. Usually the rise of the rotation speed will result in the decrease of the phase margins of the forward whirling mode, and consequently the coefficient of the controller should be adjusted with the rotor speed in order to guarantee the adequate phase margins of the different whirling modes in the expected speed range.

Simultaneously, from the root locus with respect to rotor speed from 0 to $220 \mathrm{~Hz}$, the MSR system loses its stability when the rotor speed is lower than $55 \mathrm{~Hz}$ or higher than $200 \mathrm{~Hz}$, which also agrees well with the results from the proposed method.

To further testify the correctness of the stability criterion, the corresponding experiments have been performed. For the reason that the MSR is unstable at the speed of $0 \mathrm{~Hz}$, the speed increases from $0 \mathrm{~Hz}$ to nearly $60 \mathrm{~Hz}$ by setting the cross proportion $48 \mathrm{~Hz}$. And then the MSR decreases its speed slowly with $150 \mathrm{~Hz}$ cross proportion. It can be found that the MSR loses its stability and collides with the auxiliary bearing at about $52 \mathrm{~Hz}$. Figures 8 (a) and 8(b) present, respectively, 


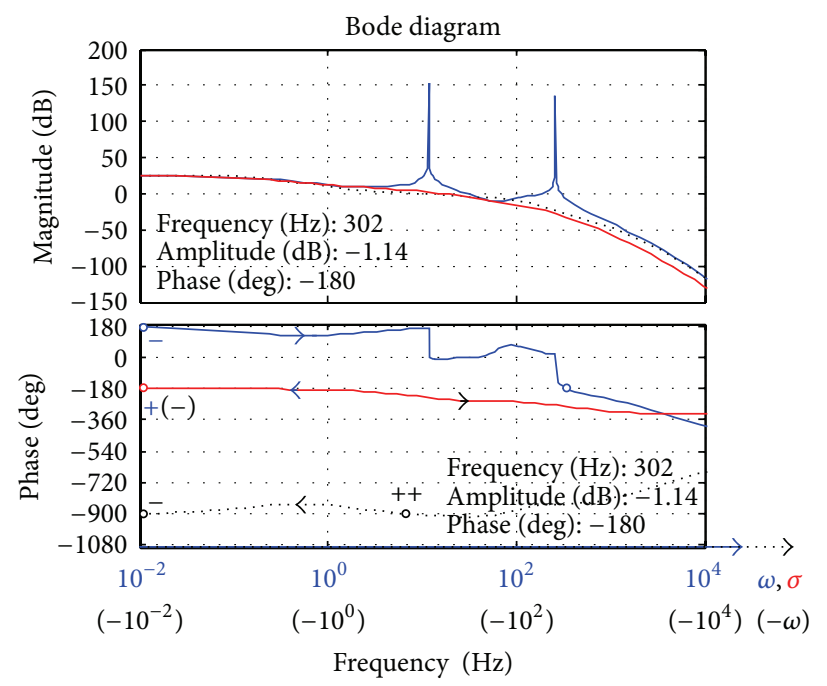

(a)

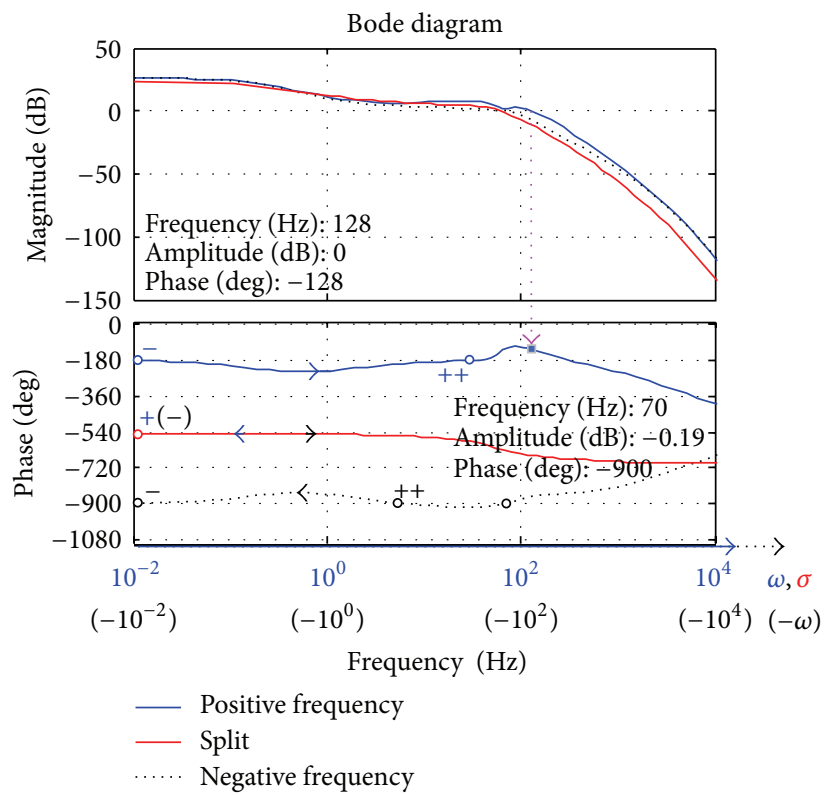

(c)

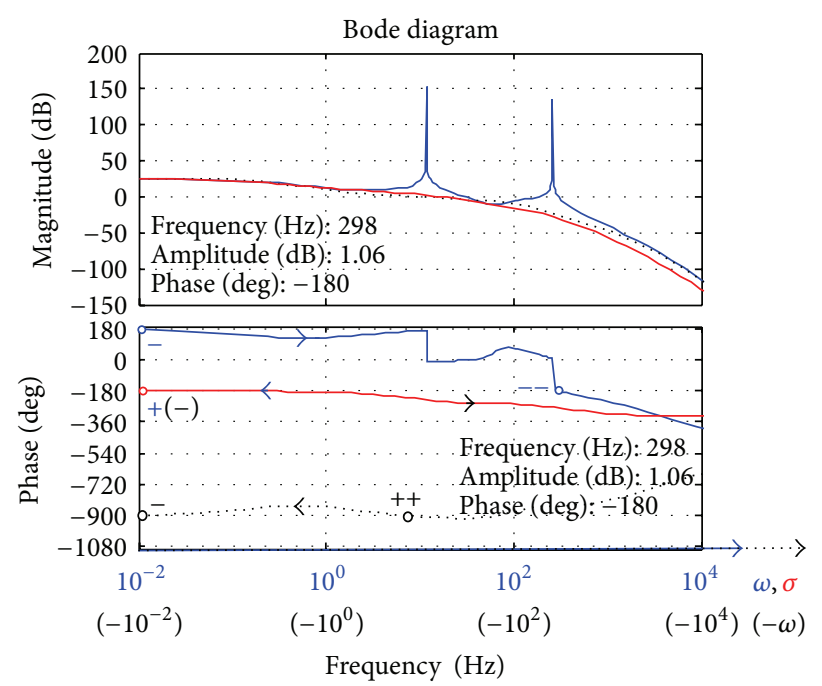

(b)

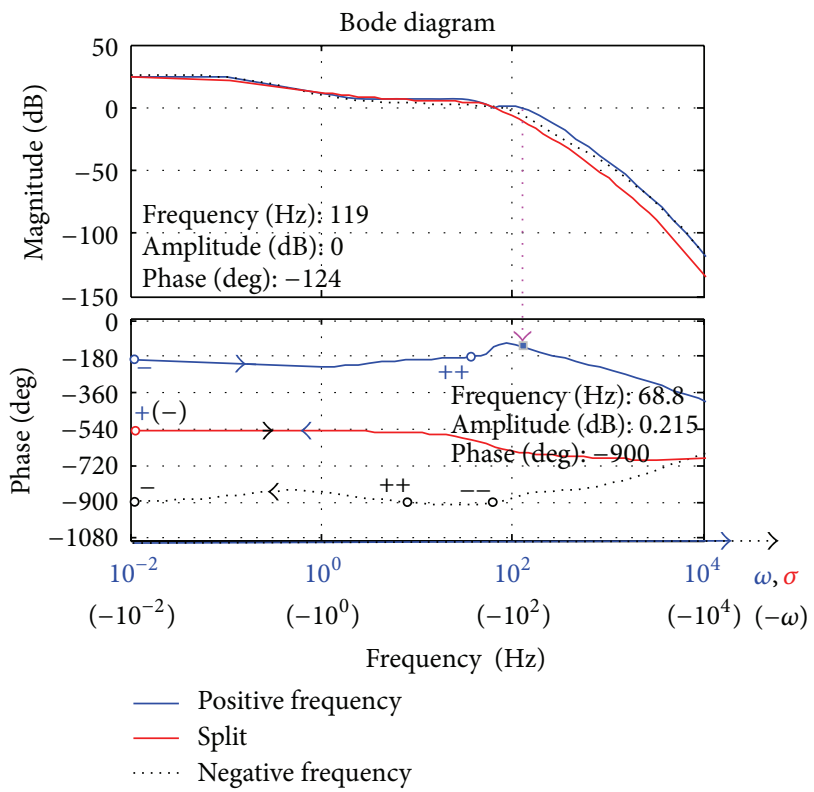

(d)

FiguRE 6: Complex-coefficient Bode diagram of the SISO system with $150 \mathrm{~Hz}$ cross proportion and different rotation speeds. (a) $200 \mathrm{~Hz}$. (b) $205 \mathrm{~Hz}$. (c) $55 \mathrm{~Hz}$. (d) $50 \mathrm{~Hz}$.

its radial displacement responses in $Y$-direction (including channels $A_{y}$ and $B_{y}$ ) and the corresponding frequency spectrum characteristics before and after the whirling mode loses its stability. Besides, the Lissajous figure of terminal $A$ of the MSR, which is generated by $h_{a x}$ and $h_{a y}$, is also tracked as shown in Figure 8(c). From Figure 8(a), it can be seen that the instability is caused by rotational mode instead of translational mode since the motion directions of channels $A_{y}$ and $B_{y}$ are opposite, which can also be testified from Figure 8(c). From Figure 8(b), the critical rotor speed is about $52 \mathrm{~Hz}$, and its corresponding whirling frequency is about $70 \mathrm{~Hz}$, which agrees well with the simulation results. Additionally, from Figure 8(c), it can be obtained that the instability mode is backward whirl instead of forward whirl since the whirling direction is opposite to the rotation.

\section{Conclusion}

To analyze directly and simply the stability of the crosscoupled two-dimensional antisymmetrical system, this paper presents a stability analysis method based on complexcoefficient frequency characteristics. Through variable reconstruction, the MIMO system is converted into a SISO system with complex coefficients. The closed-loop pole locations law and the controllability as well as observability before and after the variable reconstruction are studied and revealed, 


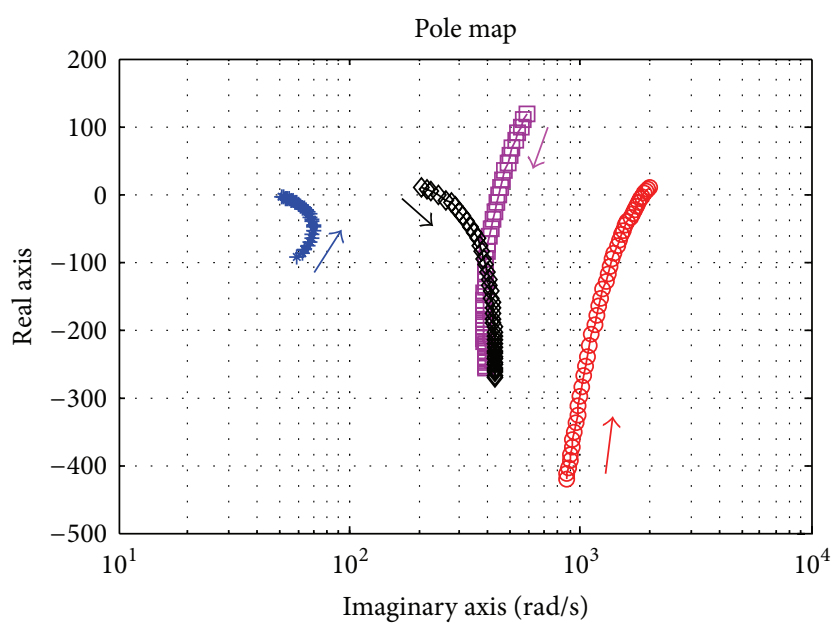

FIGURE 7: Root locus with respect to rotor speed from 0 to $220 \mathrm{~Hz}$. Note: the arrowhead denotes the increased direction of rotor speed from 0 to $150 \mathrm{~Hz}$.

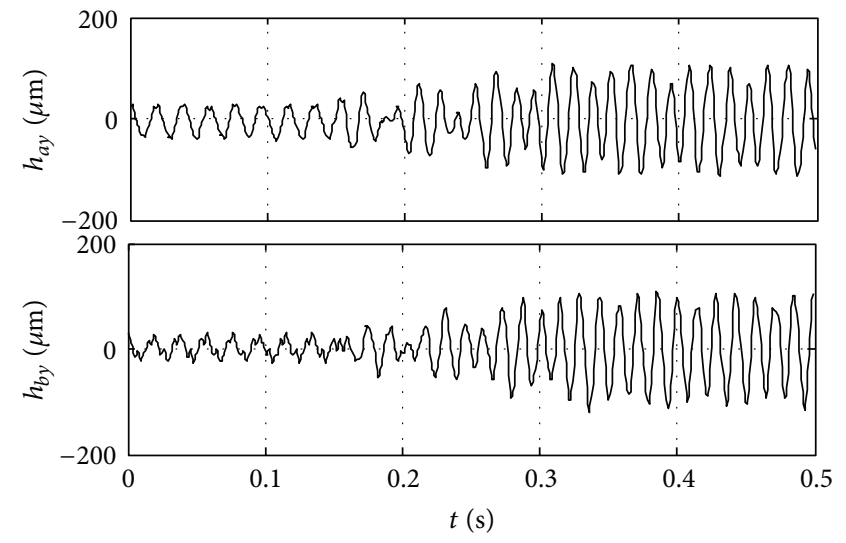

(a)

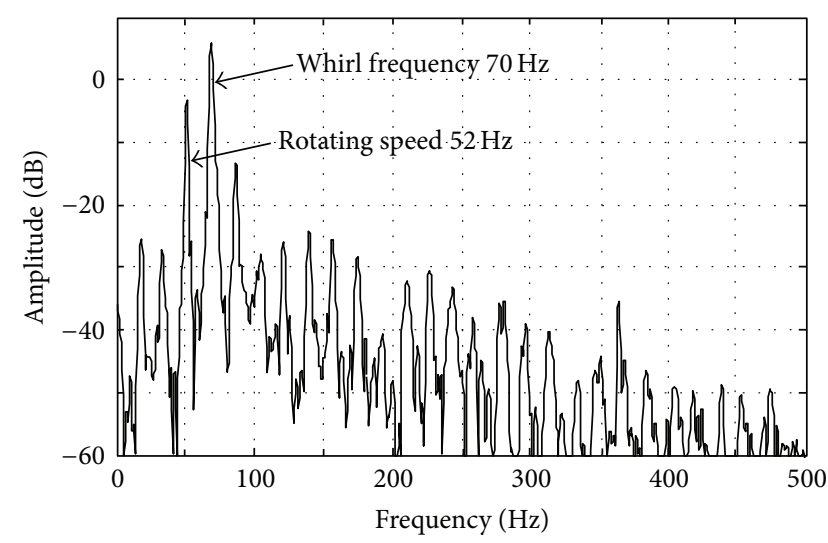

(b)

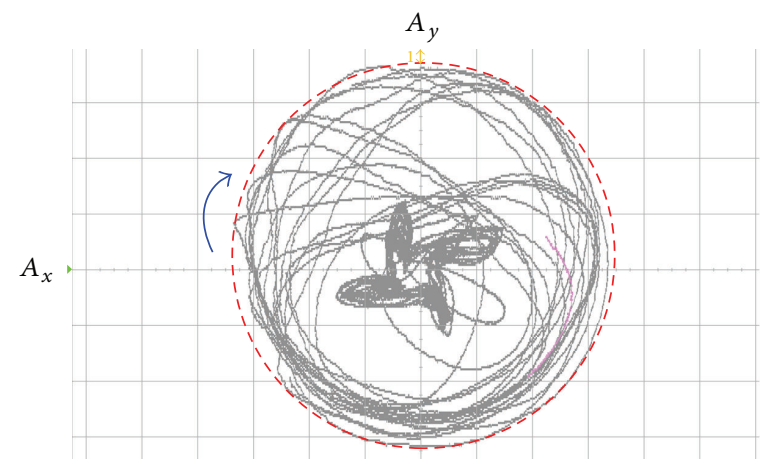

(c)

FIGURE 8: Experimental results. (a) Radial displacement responses of channels $A_{y}$ and $B_{y}$. (b) Frequency spectrum of displacement $h_{a y}$. (c) Lissajous figure of terminal $A$ of the MSR. Note: the arrowhead denotes the direction of the rotation and the circle drawn by dotted line means the auxiliary bearing.

which demonstrate that the two systems before and after variable reconstruction possess the same absolute and relative stability. Then the Nyquist stability criterion is extended from a real-coefficient system to a complex-coefficient system according to argument principle.
Combined with the rigid MSR system with significant gyroscopic effects, simulation and experimental results demonstrate that the proposed stability analysis method is feasible, correct, and quite simple to implement. Compared with the existing methods, the presented one can greatly 
simplify the analysis, design, and debugging of the control system.

\section{Appendix}

In Table $1, x_{a}$ is the radial clearance of the auxiliary bearings, that is, the protective air gaps of the ball bearings.

In Table $2, k_{a m}$ and $i_{c}$ are the proportional and feedback coefficients of the current-loop controller for the MSR system, $k_{p}, k_{i}, k_{d}$ are the proportional, integral, and differential coefficients of the basic PID controller for the MSR system; $k_{h c}$ and $k_{l c}$ are the cross coefficients of the high-pass and lowpass filters, respectively; $k_{c}$ is the total cross coefficient; $f_{h}$ and $f_{l}$ are the cut-off frequencies of the two-order high-pass and low-pass filters, respectively.

\section{Conflict of Interests}

The authors declare that there is no conflict of interests regarding the publication of this paper.

\section{Acknowledgments}

The authors are very grateful to Professor Y. Hong and Professor L. Guo from the Chinese Academy of Sciences and Professor S. S. Ge from the National University of Singapore for their valuable suggestions and instructive comments.

\section{References}

[1] T. D. Nguyen, K.-J. Tseng, S. Zhang, and H. T. Nguyen, "A novel axial flux permanent-magnet machine for flywheel energy storage system: design and analysis," IEEE Transactions on Industrial Electronics, vol. 58, no. 9, pp. 3784-3794, 2011.

[2] S. P. Bhat and P. K. Tiwari, "Controllability of spacecraft attitude using control moment gyroscopes," IEEE Transactions on Automatic Control, vol. 54, no. 3, pp. 585-590, 2009.

[3] J. Fang and Y. Ren, "High-precision control for a single-gimbal magnetically suspended control moment gyro based on inverse system method," IEEE Transactions on Industrial Electronics, vol. 58, no. 9, pp. 4331-4342, 2011.

[4] J. Fang and Y. Ren, "Decoupling control of a magnetically suspended rotor in a control moment gyro based on inverse system method," IEEE/ASME Transactions on Mechatronics, vol. 17, pp. 1133-1144, 2012.

[5] H. Bang and H. D. Choi, "Attitude control of a bias momentum satellite using moment of inertia," IEEE Transactions on Aerospace and Electronic Systems, vol. 38, no. 1, pp. 243-250, 2002.

[6] Y. Li, Z. Q. Zhu, D. Howe, C. M. Bingham, and D. A. Stone, "Improved rotor-position estimation by signal injection in brushless AC motors, accounting for cross-coupling magnetic saturation," IEEE Transactions on Industry Applications, vol. 45, no. 5, pp. 1843-1850, 2009.

[7] S. S. Broussev and N. T. Tchamov, "Quality factor analysis for cross-coupled LC oscillators using a time-varying root locus," IEEE Transactions on Circuits and Systems II, vol. 59, no. 1, pp. 25-29, 2012.

[8] F.-J. Lin, P.-H. Chou, C.-S. Chen, and Y.-S. Lin, "DSP-based cross-coupled synchronous control for dual linear motors via intelligent complementary sliding mode control," IEEE Transactions on Industrial Electronics, vol. 59, no. 2, pp. 1061-1073, 2012.

[9] H.-J. Lee, S. Jung, and S.-K. Sul, "A current controller design for current source inverter-fed AC machine drive system," IEEE Transactions on Power Electronics, vol. 28, pp. 1366-1381, 2013.

[10] M. M. Hossain, G. Lu, and Y. Yan, "Optical fiber imaging based tomographic reconstruction of burner flames," IEEE Transactions on Instrumentation and Measurement, vol. 61, no. 5, pp. 1417-1425, 2012.

[11] F. Heismann, "Improved optical compensator for first- and second-order polarization-mode dispersion," IEEE Photonics Technology Letters, vol. 17, no. 5, pp. 1016-1018, 2005.

[12] H. Mukaidani and H. Xu, "Pareto optimal strategy for stochastic weakly coupled large scale systems with state dependent system noise," IEEE Transactions on Automatic Control, vol. 54, no. 9, pp. 2244-2250, 2009.

[13] J. Rootenberg and O. J. Oso, "On cross coupling and stability in nonlinear control systems," IEEE Transactions on Automatic Control, vol. 16, no. 1, pp. 73-75, 1971.

[14] D. Newman, "The analysis of cross-coupling effects on the stability of two-dimensional, orthogonal, feedback control systems," IEEE Transactions on Automatic Control, vol. 5, pp. 314320, 1960.

[15] Z. Chen, "PI and sliding mode control of a Cuk converter," IEEE Transactions on Power Electronics, vol. 27, no. 8, pp. 3695-3703, 2012.

[16] M. Margaliot and G. Langholz, "The Routh-Hurwitz array and realization of characteristic polynomials," IEEE Transactions on Automatic Control, vol. 45, no. 12, pp. 2424-2427, 2000.

[17] A. A. El-Marhomy and N. E. Abdel-Sattar, "Stability analysis of rotor-bearing systems via Routh-Hurwitz criterion," Applied Energy, vol. 77, no. 3, pp. 287-308, 2004.

[18] M.-T. Ho, A. Datta, and S. P. Bhattacharyya, "An elementary derivation of the Routh-Hurwitz criterion," IEEE Transactions on Automatic Control, vol. 43, no. 3, pp. 405-409, 1998.

[19] C. Yang, Q. Zhang, J. Sun, and T. Chai, "Lur'e Lyapunov function and absolute stability criterion for Lur'e singularly perturbed systems," IEEE Transactions on Automatic Control, vol. 56, no. 11, pp. 2666-2671, 2011.

[20] K. S. Ray and D. D. Majumder, "Application of circle criteria for stability analysis of linear SISO and MIMO systems associated with fuzzy logic controller," IEEE Transactions on Systems, Man and Cybernetics, vol. 14, no. 2, pp. 345-349, 1984.

[21] M. Fardad and B. Bamieh, "An extension of the argument principle and nyquist criterion to a class of systems with unbounded," IEEE Transactions on Automatic Control, vol. 53, no. 1, pp. 379-384, 2008.

[22] R. Turner, S. Walton, and R. Duke, "A case study on the application of the Nyquist stability criterion as applied to interconnected loads and sources on grids," IEEE Transactions on Industrial Electronics, vol. 60, no. 6, pp. 2740-2749, 2013.

[23] A. G. Yepes, F. D. Freijedo, Ó. López, and J. Doval-Gandoy, "Analysis and design of resonant current controllers for voltagesource converters by means of nyquist diagrams and sensitivity function," IEEE Transactions on Industrial Electronics, vol. 58, no. 11, pp. 5231-5250, 2011.

[24] J. Kim, D. G. Bates, and I. Postlethwaite, "Evaluation of stochastic effects on biomolecular networks using the generalized nyquist stability criterion," IEEE Transactions on Automatic Control, vol. 53, no. 8, pp. 1937-1941, 2008. 
[25] F. Fontana, "Use of the Nyquist stability criterion in the design of interactive audio digital filters," IEEE Signal Processing Letters, vol. 18, no. 4, pp. 271-274, 2011.

[26] M. Vidyasagar, R. K. Bertschmann, and C. S. Sallaberger, "Some simplifications of the graphical Nyquist criterion," IEEE Transactions on Automatic Control, vol. 33, no. 3, pp. 301-305, 1988.

[27] J. Fotakis, M. J. Grimble, and B. Kouvaritakis, "A comparison of characteristic locus and optimal designs for dynamic ship positioning systems," IEEE Transactions on Automatic Control, vol. 27, no. 6, pp. 1143-1157, 1982.

[28] K. S. Ray and B. A. Kouvaritakis, "An extension of the characteristic sequences method to the case of repeated roots," IEEE Transactions on Automatic Control, vol. 29, no. 10, pp. 933-934, 1984.

[29] M. N. Saadatzi, J. Poshtan, and M. H. Saadatzi, "Optimal multivariable two-degree-of-freedom control of electric wheelchair using non-dominated sorting genetic algorithm-II," in Proceedings of the 20th IEEE International Conference on Control Applications (CCA '11), pp. 376-381, September 2011.

[30] Yang, Q. Guan, P. X. Wang, and X. Zhang, "Stability analysis of matrix converter with constant power loads and LC input filter," in Proceedings of the 7th International Power Electronics and Motion Control Conference (IPEMC '12), pp. 900-904, 2012.

[31] R. S. Baheti, "Multivariable frequency domain controller for magnetic suspension and balance systems," IEEE Transactions on Automatic Control, vol. 29, no. 8, pp. 725-728, 1984.

[32] O. D. I. Nwokah, R. E. Nordgren, and G. S. Grewal, "Inverse Nyquist array: a quantitative theory," IEE Proceedings: Control Theory and Applications, vol. 142, no. 1, pp. 23-30, 1995.

[33] A. Postlethwaite and G. J. MacFarlane, A Complex Variable Approach to the Analysis of Linear Multivariable Feedback Systems, Springer, New York, NY, USA, 1979.

[34] Y. Okada, B. Nagai, and T. Shimane, "Cross-feedback stabilization of the digitally controlled magnetic bearing," Journal of Vibration and Acoustics, vol. 114, no. 1, pp. 54-59, 1992.

[35] M. Ahrens, L. Kučera, and R. Larsonneur, "Performance of a magnetically suspended flywheel energy storage device," IEEE Transactions on Control Systems Technology, vol. 4, no. 5, pp. 494-502, 1996.

[36] G. V. Brown, A. Kascak, R. H. Jansen, T. P. Dever, and K. P. Duffy, "Stabilizing gyroscopic modes in magnetic-bearing-supported flywheels by using cross-axis proportional gains," in AIAA Guidance, Navigation, and Control Conference, pp. 1132-1143, San Francisco, Calif, USA, August 2005, AIAA 2005-5955.

[37] A. F. Kascak, G. V. Brown, R. H. Jansen, and T. P. Dever, "Stability limits of a PD controller for a flywheel supported on rigid rotor and magnetic bearings," in AIAA Guidance, Navigation, and Control Conference, pp. 1144-1155, San Francisco, Calif, USA, August 2005, AIAA 2005-5956.

[38] A. A. El-Marhomy, "Effect of various rotor-bearing system parameters on the whirl stability of system rotational modes of motion," Energy Conversion and Management, vol. 38, no. 1, pp. 73-82, 1997.

[39] F. Sorge and M. Cammalleri, "Control of hysteretic instability in rotating machinery by elastic suspension systems subject to dry and viscous friction," Journal of Sound and Vibration, vol. 329, no. 10, pp. 1686-1701, 2010.

[40] S. E. Mushi, Z. Lin, and P. E. Allaire, "Design, construction, and modeling of a flexible rotor active magnetic bearing test rig," IEEE/ASME Transactions on Mechatronics, vol. 17, pp. 1170-1182, 2008.
[41] Y. Ren, D. Su, and J. Fang, "Whirling modes stability criterion for a magnetically suspended flywheel rotor with significant gyroscopic effects and bending modes," IEEE Transactions on Power Electronics, vol. 28, pp. 5890-5901, 2013.

[42] Y. Ren and J. Fang, "Current-sensing resistor design to include current derivative in PWM H-bridge unipolar switching power amplifiers for magnetic bearings," IEEE Transactions on Industrial Electronics, vol. 59, pp. 4590-4600, 2012.

[43] K. S. Yeung and W. T. Wong, "Root-locus plot of systems with time delay," Electronics Letters, vol. 18, no. 11, pp. 480-481, 1982.

[44] J. Fang, Y. Ren, and Y. Fan, "Nutation and precession stability criterion of magnetically suspended rigid rotors with gyroscopic effects based on positive and negative frequency characteristics," IEEE Transactions on Industrial Electronics, vol. 61, pp. 2003-2014, 2014.

[45] U. T. Jonsson and C.-Y. Kao, "Consensus of heterogeneous LTI agents," IEEE Transactions on Automatic Control, vol. 57, no. 8, pp. 2133-2138, 2012. 


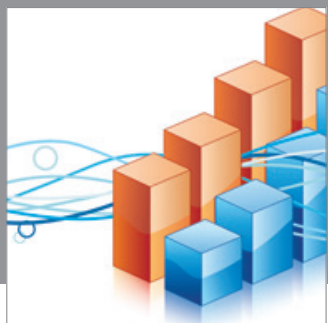

Advances in

Operations Research

mansans

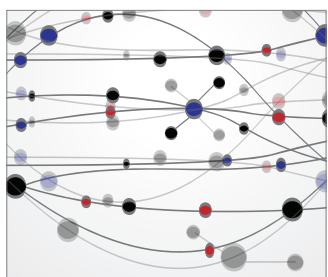

The Scientific World Journal
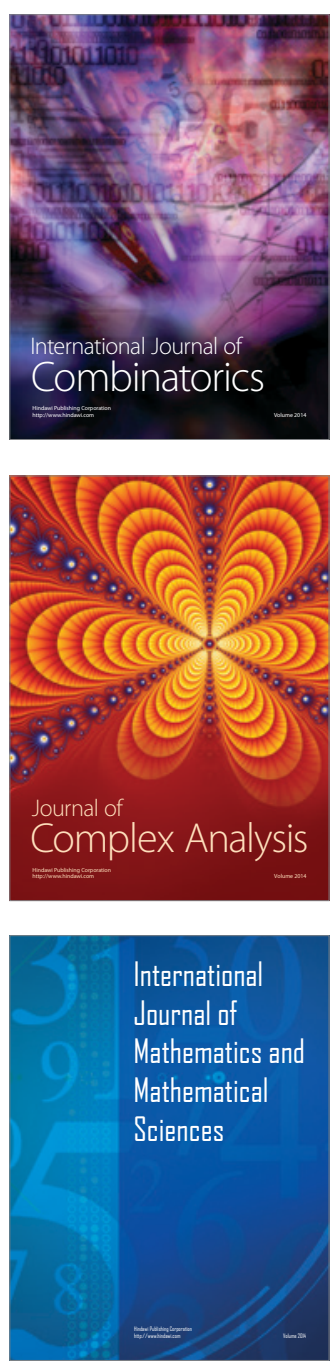
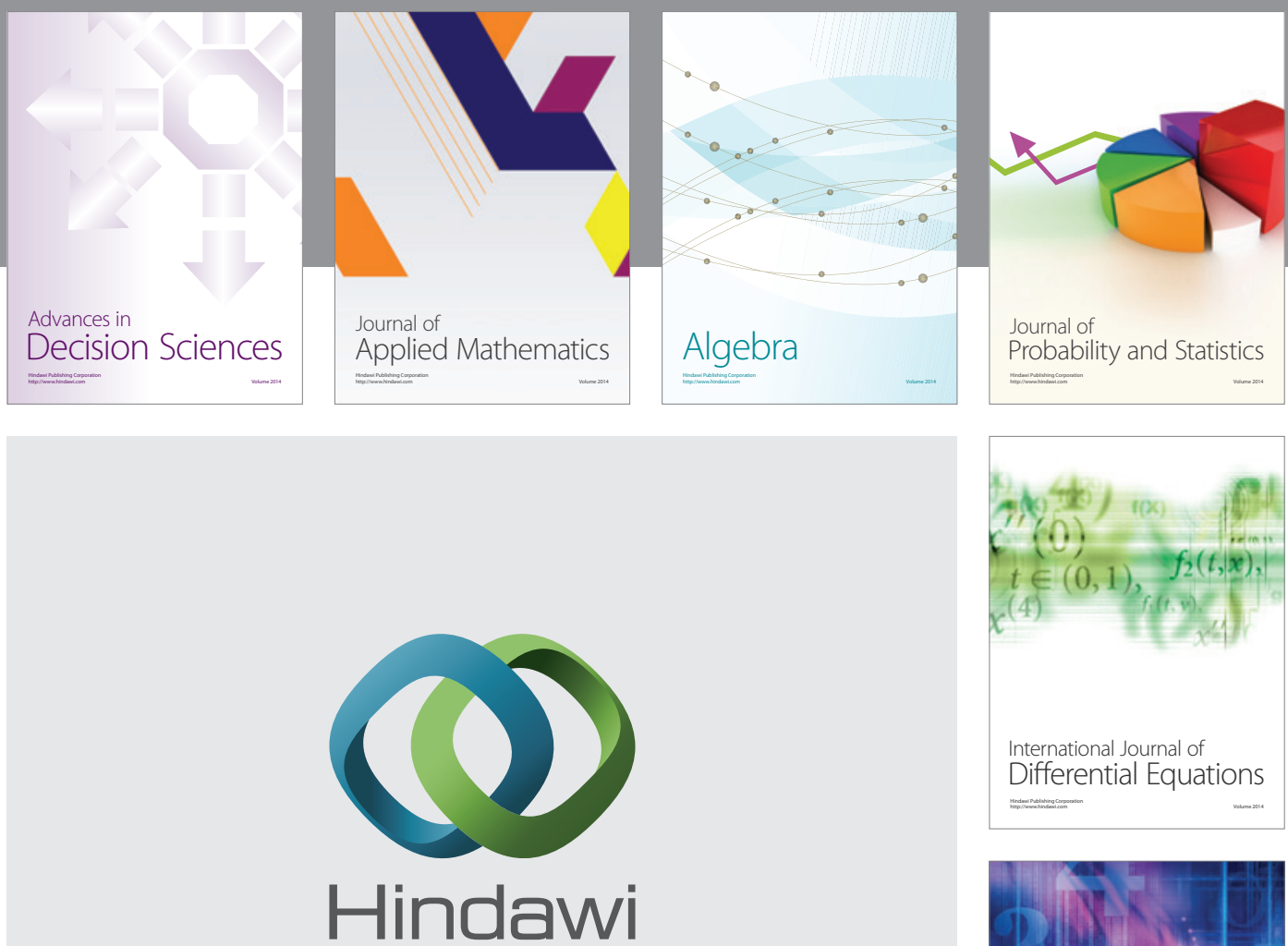

Submit your manuscripts at http://www.hindawi.com
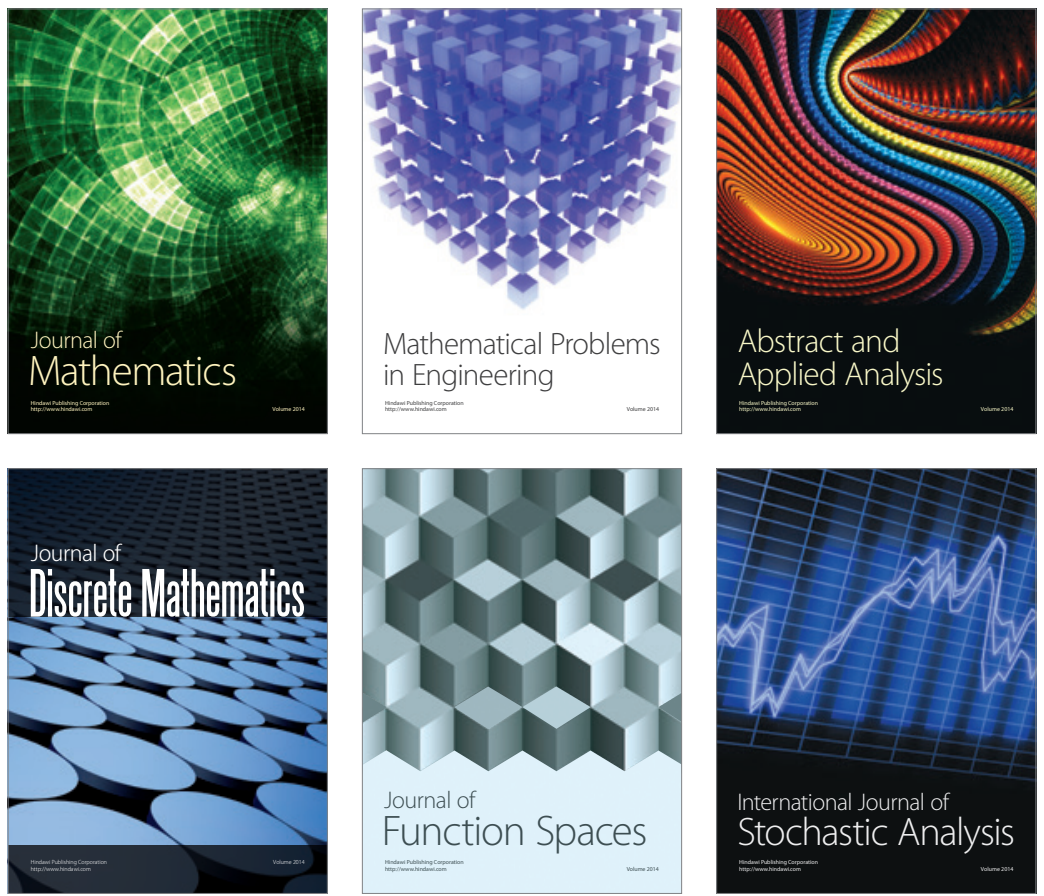

Journal of

Function Spaces

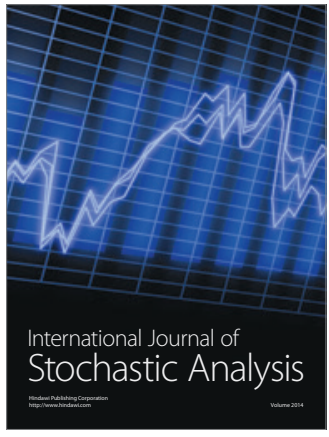

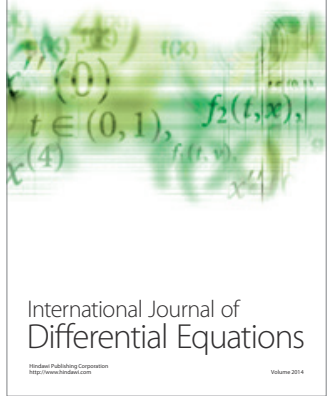
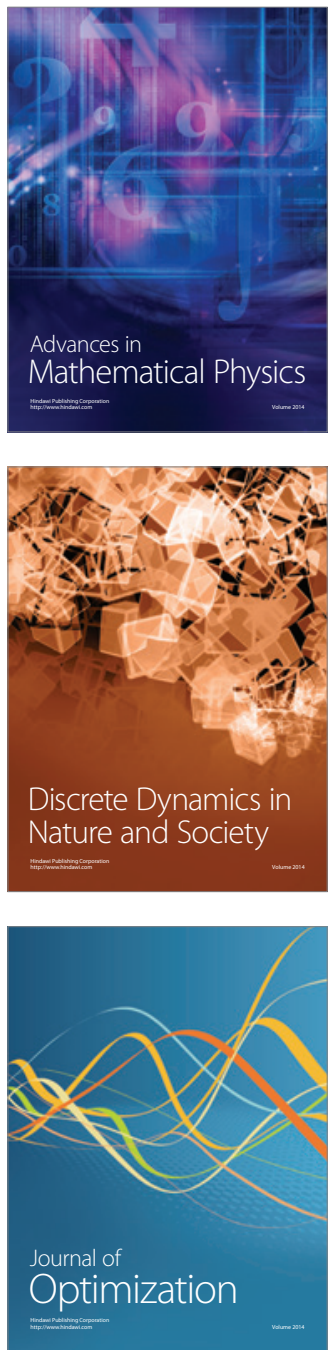